\section{Zervikales Kopfweh - Science oder Fiction?}

\author{
R. Agosti
}

Zwischen Kopfweh und Nackenschmerzen bestehen ein Fülle von Assoziationen. In zahlreichen Bedingungen gehören Nacken- und Kopfschmerzen fast unzertrennbar zusammen wie beim Schleudertrauma der Halswirbelsäule, bei Meningitis, Karotis- und Vertebralisdissektion, Neuritis des Nervus occzipitalis major (NOM). Bestens bekannt, doch wenig verstanden, ist eine idiopathische Konstellation von gleichzeitig auftretenden Kopf- und Nackenschmerzen, oft chronifizierend, welche meist "zervikozephales Syndrom» genannt wird. Nackenschmerzen können gelegentlich zeitlich hoch korrelierend als Begleitsymptom von Migräneattacken auftreten. Ob Strukturen der Halswirbelsäule für die Entstehung von Kopfschmerzen oder Migräne verantwortlich sein könnten, wird seit sehr langer Zeit kontrovers diskutiert, und die Diskussion soll hier erneut beleuchtet werden, da sie nichts von ihrer Aktualität - aber auch nichts von ihrer Verworrenheit - eingebüsst hat. Kopfschmerzen, durch Manipulationen am Nacken ausgelöst, werden von verschiedenen Autoren als "zervikogene Kopfschmerzen" bezeichnet. Falls diese sehr spezifische Konstellation tatsächlich eine Entität repräsentiert, könnten Hinweise für die Pathogenese von Migräne und Kopfschmerzen daraus resultieren.

Seit Mitte des 18. Jahrhunderts sind verschiedene Persönlichkeiten mit der Diskussion um den hypothetischen zervikal ausgelösten Kopfschmerz verbunden: Schützenberger (1853), Barré-Lieou (1925), Bärtschi-Rochaix (1943) und einige andere haben versucht, mit mehr oder weniger Überzeugungskraft ein eigenständiges Krankheitsbild abzugrenzen. Dies ist schwierig, da Kopf- und Nackenschmerzen oft mit verblüffend ähnlichen Symptomen assoziiert sind und sehr häufig gleichzeitig auftreten. Auf diese Schwierigkeit deutet auch die lange (nicht abschliessende) Auflistung von Synonymen hin: okzipitale Neuralgie, Myalgie-Neuralgie, spondylogenes Kopfweh, «migraine cervicale», Barré-Lieou-Syndrom. In den letzten 15 Jahren hat vor allem der norwegische Neurologe Sjaastad versucht, mittels einer neuen verbindlichen Definition das zervikogene Kopfweh in den Griff zu bekommen.

Korrespondenz:

Dr. med. Reto Agosti

Universitätsspital Zürich

Neurologie

Abteilung für Kopfweh und -schmerz

CH-8091 Zürich
Zahlreiche potentiell schmerzerzeugende Strukturen finden sich am Nacken: Nerven mit zugehörigen Wurzeln und Ganglien, Facettgelenke mit Kapseln, Bandscheiben, Periost, Sehnen und Bänder sowie arterielle und venöse Gefässe. Besondere Aufmerksamkeit geniessen die Facettgelenke, die durch Irritationen (z.B. Aufdehnung durch gezielte Kontrastmittelinjektionen) charakteristische topographische Schmerzprojektionen in Kopf, Nacken und Schultern hervorrufen können. Die Irritationszone nach C2/3Stimulation ist ipsilateral hoch nuchal und am Hinterhaupt, während die C3/4- bis C6/7-Zonen allesamt unterhalb des Kopfes liegen [1]. Zudem sind die Nervi occzipitales major und minor sowie medius (der sogenannte "third occipital nerve») oft Gegenstand von Untersuchungen und Therapieansätzen (siehe unten). Es ist ein weitverbreitetes Missverständnis, dass die zervikalen Kopfschmerzen auf pathologische Veränderungen der knöchernen Strukturen und Bandscheiben allein zurückzuführen sind. Radiologische Abklärungen sind nur indiziert, wenn Anamnese und körperliche Untersuchung den Verdacht auf Läsionen des Nervensystems oder Bewegungsapparates oder anderer Gewebe ergibt. Pathophysiologisch interessant ist die Konvergenz von zervikalen und zephalen Schmerzafferenzen in den Trigeminuskernen sowie deren physiologisch gesehen zentrale Lage im Hirnstamm, wo sich Regelkreise, die bei Migräne eine Rolle spielen, finden und aktiviert werden. Das trigemino-vaskuläre System hat eine wichtige Stellung in der Entstehung von Kopf- und Nackenschmerzen.

Die heutige Kontroverse dreht sich meist um die erstmals 1983 von Sjaastad publizierten diagnostischen Kriterien für "zervikogenes Kopfweh", welche 1990 und 1998 [2] revidiert wurden. Dabei verwendet er drei Hauptkriterien sowie einige nicht obligate zusätzliche Kopfweh- und Nebenkriterien.

Die Hauptkriterien verlangen

1. Kopfschmerzen, welche durch Kopfbewegungen, Kopfhaltungen oder durch Druck auf Nackenstrukturen ausgelöst werden müssen,

2. strikt einseitige Kopfschmerzen ohne Seitenwechsel, und

3. (zumindest für wissenschaftliche Arbeiten gefordertes) Ansprechen der Schmerzen auf anästhesiologische Blockade.

Die Kopfschmerzen sollen mittelschwer, nicht pulsierend und nicht stechend, vom Nacken ausgehend, episodisch mit variabler Dauer oder aber fluktuierend-kontinuierlich sein. Eine ähnliche Definition bietet die "International Association for the Study of Pain" [3] an. Die IASP verlangt auch keine spezifische Nackenpathologie für die Diagnose, jedoch ebenso das Verschwinden der Kopfschmerzen auf NOM- oder C2/3-Blockade. Der Kriterienkatalog der "International Headache Society" [4] offeriert lediglich die Kategorie "Kopfweh assoziiert mit Nackenerkrankungen», in die allfällige zervikogene Kopfschmerzen fallen. Allerdings fordert die IHS radiologische Veränderungen, z. B. eingeschränkte Beweglichkeit, wobei aber spondylotische Anzeichen nicht zählen. 
Neben den Hauptkriterien wird eine nichtobligate Liste von eher beschreibenden Nebenkriterien angeboten: eher weibliches Geschlecht, geringer oder fehlender Effekt von Indometacin, Ergotamin oder Sumatriptan auf Kopf- und Nackenschmerzen. HWS Schleudertraumen oder Schädelhirntraumen werden oft in der Anamnese genannt. Noch vager wird die Abgrenzung von anderen Kopfschmerzen durch den Umstand, dass auch Migränesymptome wie Nausea, Photo- und Phonophobie, Schwindel und Verschwommensehen sowie Schluckschwierigkeiten assoziiert sein können.

Strenge Einseitigkeit der Kopfschmerzen findet man gemäss einer Untersuchung von Leone [5] am häufigsten in einer Gruppe von (gemäss IHS) nicht klassifizierbaren primären Kopfschmerzen und am zweithäufigsten unter Migränepatienten. Wenn die so gefundenen Patientenzahlen weiterhin durch die geforderte Kopfschmerzauslösung durch Nackenmanipulationen eingegrenzt werden, erfüllen gerade noch $0,4 \%$ die Sjaastad-Kriterien für zervikogenes Kopfweh. Es existieren zudem keine klaren Kriterien zur Unterscheidung von zervikogenen und myofaszialen Triggerpunkten.

Das Ansprechen auf zervikale Schmerzblockaden, hauptsächlich von NOM und C2/3-Gelenk, wird in der Literatur mit sehr unterschiedlichen Häufigkeiten berichtet, z.B. $6 \%$ bis $85 \%$ bei Migränen, $71 \%$ bei posttraumatischen Kopfschmerzen und $14 \%$ bei Spannungskopfschmerzen. Durch die bei Irritation des C2/3-Gelenkes relativ konsistente Schmerzausstrahlung in den Kopf, sind C2/3-Blockaden ein auf der Hand liegendes Ziel von Kopf- und Nackenschmerzbehandlungen; leider auch mit sehr unterschiedlichen Resultaten. Gemäss einer doppelblinden neueren Publikation über Schmerzbehandlung mittels Facettblöcken wurde gerade der C2/3-Zugang als besonders schwierig beschrieben und deswegen nicht untersucht [6]. Unklarheit besteht unter anderem auch darüber, wie zuverlässig die injizierten Lokalanästhetika im Facettgelenk intraartikulär plaziert werden können und zu welchem Grad die umgebenden Strukturen mitanästhesiert werden.

Differentialdiagnostisch kommen fast alle primären Kopfwehtypen in Frage: Migräne mit und ohne Aura, Spannungskopfweh, Clusterkopfweh, chronisch paroxysmale Hemikranie (CPH), Hemicrania continua, atypischer Gesichtsschmerz, Okzipitalisneuralgie, Gefässdissektionen sowie Schleudertrauma und andere posttraumatische Kopfschmerzen. Beim Clusterkopfweh finden sich ipsilateral zum («unermesslich intensiven") Schmerz auch noch sympathische oder parasympathische Dysfunktionen und bei der Dissektion oftmals ein ipsilaterales Hornersyndrom sowie Strömungsgeräusche. Die Okzipitalisneuralgie ist charakterisiert durch Schmerzverteilung im Innervationsgebiet des NOM und Schmerzprovokation durch Palpation der Nerven, besonders subokzipital.

Zur Therapie der zervikalen Kopfschmerzen und des zervikozephalen Syndromes fehlen gute kontrollierte Studien [7], allein schon wegen der Klassifikationsproblematik. Da ein starkes Überlappen zu an- deren primären Kopfschmerzen besteht, sind ähnliche Therapiestrategien wie für diese im Einsatz, d.h. NSARs und Migränemedikamente (inklusive Triptane) für den Akutfall und v.a. trizyklische Antidepressiva (TCAs) für den chronischen Schmerz. Erste Wahl ist meist Amitriptylin (10 bis $75 \mathrm{mg}$ abends), das nicht zuletzt durch seine schlafanstossende Eigenschaft von Nutzen sein kann. Stimulierende TCAs sowie evtl. Serotoninwiederaufnahmehemmer können ebenfalls brauchbar sein. Im weiteren können Antiepileptika wie Valproat, Carbamazepin, Gabapentin und andere versucht werden. Betablocker und Kalziumantagonisten sollten in Betracht gezogen werden, wenn migränoide Symptome, Hypertonie oder eine migränepositive Familienanamnese vorhanden sind. Lokal können Antirheumatika im Okklusivverfahren oder Capsaicin (initial $5 \times$ täglich) angewendet werden.

Physikalische Therapien haben eine feste Stellung in der Behandlung, obwohl es durch zwei kürzliche Studien zu einer Pattsituation gekommen ist, nachdem diese Studien zeigten, dass "Nichtstun" besser war für Schmerzen nach Schleudertrauma als physikalische Behandlungen. Diese Resultate stehen im Gegensatz zu weitverbreiteter guter klinischer Erfahrung mit Physiotherapie und verwandten Verfahren. Anzumerken ist, dass auch in diesen Studien nicht wenige Patienten auf physikalische Methoden ansprachen. Eine alleinseligmachende Methode gibt es sicherlich nicht, aber die gründliche Abklärung und Aufklärung, Beleuchtung des psychosozialen Konstellation, das Ernstnehmen der Patienten und deren Motivation zum Durchhalten tragen Entscheidendes zum Erfolg bei. Zervikale Kopfschmerzen sind oft transient, wenn auch in längerem zeitlichem Rahmen. Zudem muss zu äusserster Zurückhaltung gegenüber chirurgischen Eingriffen ermahnt werden. Zahlreiche Verfahren waren einst als hochwirksam angesehen und sind danach dennoch verschwunden. Beispielhaft sei die "Exhaerese» des NOM erwähnt, ein Verfahren, in dem der Nerv durchtrennt und aus seinem Bett gezogen wird. Ein kürzlich von mir betreuter Patient hat nach Exhaerese heute nicht nur erneut bilaterale subokzipitale und zephale Schmerzen, sondern auch wieder Sensibilität im Innervationsgebiet der entfernten Nerven. Die C2/3-Blockade hat lediglich diagnostischen Wert und ist technisch schwierig einwandfrei durchzuführen. Andererseits seien auch Berichte und Beobachtungen erwähnt, nach denen wiederholte Blockaden mit Lokalanästhetika zu bleibendem Erfolg führen können.

Zusammenfassend sei erwähnt, dass trotz einiger Arbeiten zu Klassifikation und Pathophysiologie und sehr vielen Studien und Berichten zur Behandlung von zervikalen Schmerzen keine schlüssigen Daten vorliegen. Insbesondere fehlen einwandfreie, kontrollierte Studien zur Therapie, von einigen wenigen Ausnahmen abgesehen. Der hypothetische zervikogene Kopfschmerz kann nicht schlüssig als Entität von primären Kopfschmerzen mit Nackenbeteiligung abgegrenzt werden. Eine Korrelation mit degenerativen Nackenveränderungen, insbesondere anhand radiologischer Darstellung, ist nicht gegeben. Thera- 
peutisch sind Massnahmen, die auch bei primären Kopfschmerzen wirksam sind, am erfolgversprechendsten. Chirurgische Eingriffe sind in den seltensten Fällen indiziert.

\section{Literatur}

1 Dwyer A, Aprill C, Bogduk N. Cervical zygapophyseal joint pain patterns. I: A study in normal volunteers. Spine 1990; 15:453-7.

2 Sjaastad 0, Fredriksen TA, Pfaffenrath V. Cervicogenic headache: diagnostic criteria. Headache 1998;38:442-5.

3 International Association for the Study of Pain (IASP).

Cervicogenic Headache. In: Merskey H, Bogduk N (eds.).

Classification of chronic pain. Descriptions of chronic pain syndromes and definitions of pain terms. 2nd edition. Seattle: IASP Press; 1994. p. 94-95.
4 International Headache Society (IHS), Headache Classification Committee of the International Headache Society. Classification and diagnostic criteria for headache disorders, cranial neuralgias, and facial pain. Cephalalgia 1988;8.

5 Leone M, D’Amico D, Grazzi L, Attanasio A, Bussone G. Cervicogenic headache: a critical review of the current diagnostic criteria. Pain 1998;78:1-5.

6 Lord S, Barnsley L, Wallis BJ, McDonald GJ, Bogduk N. Percutaneous radio-frequency neurotomy for chronic cervical zygapophyseal-joint pain. N Engl J Med 1996;335:1721-64.

7 Pöllmann W, Keidel M, Pfaffenrath V. Headache and the cervical spine: a critical review. Cephalalgia 1997;17:561-72. 